\title{
Comparative Study between Surgical Intervention and Endoscopic Stenting for Management of Leakage after Sleeve Gastrectomy
}

\author{
Heba Tharwat Abd El Aziz, MD; ${ }^{1}$ Mahmoud Abdelbaky Mahmoud, MD² \\ ${ }^{1}$ Department of General Surgery, Faculty of Girls, Al Azhar University, Egypt \\ ${ }^{2}$ Department of General Surgery, Faculty of Medicine, Ain Shams University, Egypt
}

Objectives: To evaluate surgical intervention versus stents insertion in management of staple line leaks after laparoscopic sleeve gastrectomy in morbidly obese patients.

Patients and methods: Prospective randomized study including 30 hemodynamically stable patients presented early post sleeve leaks discovered by routine postoperative imaging or during follow up period. Patients recruited from El-Demerdash university hospital and El Zahraa university hospital during period January 2014 to December 2018. Patients were divided in two equal ratio groups: Endoscopy group: included 15 patients post sleeve leakage underwent stent insertion and Surgery group: included 15 patients post sleeve leakage underwent surgical management.

Results: Mean interval between Initial surgery and leak in endoscopy group was $4.5 \pm 2.7$ days with most common site of leak GE junction (80\%). Mean interval between Initial surgery and leak in surgery group was $4.6 \pm 2.2$ days; while most common site leak, GE junction (73.3\%) with no statistical significance. Leak closure was achieved in 13 (86.66\%) in endoscopy group and only 2 patients required conversion $\mathrm{R}$ en $\mathrm{Y}$ with closure of leak and leak control within $3.3 \pm 5.5$ days. Average hospital stay was $8.3 \pm 4.4$ days. Leak closure achieved in $7(46.6 \%) 13(86.66 \%)$ in surgery group, seven patients required conversion to $\mathrm{R}$ en $\mathrm{Y}$ either from the start or after failure of direct suturing to control spillage, while one patients died. interval between closure of leak and leak control was $5 \pm 4.3$ days. With average hospital stay $8.3 \pm 4.4$ days. There was no statistically significant association between type of management and outcomes $(p=0.51)$. In contrary, there was statistically significant association between type of management and interval for leak control $(p<0.001)$ and hospital stay $(p=0.012)$. There was no statistically significant association between the site of leak in both groups $(p=0.48)$.

Conclusion: Stenting is a better choice for spillage patients who are essentially steady. It is less dangerous than surgical procedure.

\section{Introduction}

Laparoscopic sleeve gastrectomy (LSG) has been championed as a safe and less-invasive bariatric procedure. Compared with the Roux en-Y gastric bypass the rate of devastating complications is relatively low. There are 4 sleeve-specific perioperative complications include staple line breaks, bleeding, stenosis, and portal vein thrombosis (PVT). ${ }^{1}$

Sleeve gastrectomy is increasingly performed for the surgical management of obesity. When a surgical drain is in place, these leaks are recognized early. In the absence of a drain, the presentation is usually that of an intra-abdominal abscess, which can be drained percutaneously. Debridement of abscess cavities can be performed by passing an endoscope through the fistula, and plastic pigtail stents can be placed transmurally into the cavity to provide internal abscesses drainage with subsequent closure of the anastomotic leak site. If the leak is not closed early, the leak can then lead to a fistula to the skin at the site of the drain. Thus, early closure can prevent delayed fistula formation. A variety of endoscopic techniques can be used to close the leaks after both of these bariatric procedures. These include the use of ablation using argon plasma, clips, fibrin glue, stent placement (both SEMS and internal plastic stents through the leak), balloon dilation of the sleeve downstream from the leak, suturing devices, and tissue sealants. ${ }^{2}$ Disrupted staple line is one of the more feared complications after LSG. Leaks incidence after sleeve gastrectomy is $2-3 \%$, most of it occurring after discharge from the hospital. Extravasation of contrast most frequently along the superior one-third of the gastric staple line. Gastric leak is frequently not visible at imaging during the early postoperative period for up to 3 or 4 days, and even beyond. ${ }^{3}$ Causes of leaks may include poor surgical technique, staple line failure, and a distal gastric obstruction which is the most common cause, that causes increased intragastric pressure leading to disruption of the staple line near the gastroesophgeal junction. ${ }^{4}$ Usually patients present with tachycardia, worsening abdominal pain, fevers and chills. When postoperative patients present with these symptoms, surgeons must raise a high index of suspicion. ${ }^{5}$ The diagnostic test of choice is computed tomography (CT) with oral contrast, with a sensitivity (84\%) greater than studies with gastrografin swallow $(50 \%){ }^{6}$ The definitive algorithm of management of leaks after sleeve gastrectomy remains dependent on the 
hemodynamic stability of the patient. Unstable patients should be explored either open or laparoscopically aiming primarily at controlling the area of leakage with drains and secondly assessing the gastric tissue for debridement or repair. Most simple repairs unfortunately tend to fail, but prompt interference and control of sepsis often buys time for a more definitive solution. Stable patients can be drained using interventional radiologic techniques and should be maintained on broad-spectrum antibiotics with no oral feeding for at least 2 weeks, after that they can be reassessed radiographically for fistula healing. Patients with persistent leaks should be transferred to a tertiary bariatric center, as more treatment options include endoscopic stent placement, ${ }^{7}$ intragastric drainage, or a RouX-en-Y fistulojejunostomy or even a total gastrectomy. ${ }^{8}$ Although anastomotic leaks are rare; $1.7 \%$ to $4 \%$ in most of studies, the current rapid growth in bariatric surgery has resulted in an increase in the absolute number of patients with anastomotic fistulas. ${ }^{9}$ The mortality rates ranging from $8 \%$ to $37.5 \%$ after these leaks. ${ }^{10}$

Ultrasound imaging has little value in such conditions as it can't recognize variations from norm since heftiness and little size walled off accumulations in sub-diaphragmatic area are difficult to be visualized. ${ }^{11}$

The total appraisal chest X-ray images help ruling out different causes tachycardia such pneumonia, aspiration, embolism, or pleural affection. ${ }^{12}$

\section{Aim of the work}

To compare surgical intervention and endoscopic stenting for treatment of gastric leakage after sleeve gastrectomy in morbidly obese individuals.

\section{Patints and methods}

The present prospective randomized, study included 30 patients presented early post sleeve leaks discovered by routine postoperative imaging or during follow up period. Patients recruited from El-Demerdash university hospital and El Zahraa university hospital during period January 2014 to December 2018. A high index suspicion was obtained if there is SIRS \{unexplained tachycardia $>100 / \mathrm{min}-$ fever $\left.>38^{\circ} \mathrm{C}\right\}$, Abdominal pain and tenderness either diffuse or localized upper abdominal or Pulmonary symptoms including cough, expectoration or hiccough. The study incorporated hemodynamically stable type I spillage. Patients were divided in two equal ratio groups:

1. Endoscopy group: included 15 patients post sleeve leakage underwent stent insertion.

2. Surgery group: included 15 patients post sleeve leakage underwent surgical management.
Unstable cases displayed septic manifestations or peritonitis were excluded from the study. All patients incorporated into study were exposed to proper evaluation including: History taking including age and sex, Weight and BMI, General and Abdominal examination, Routine laboratory investigations and CT abdomen with oral and IV contrast were done.

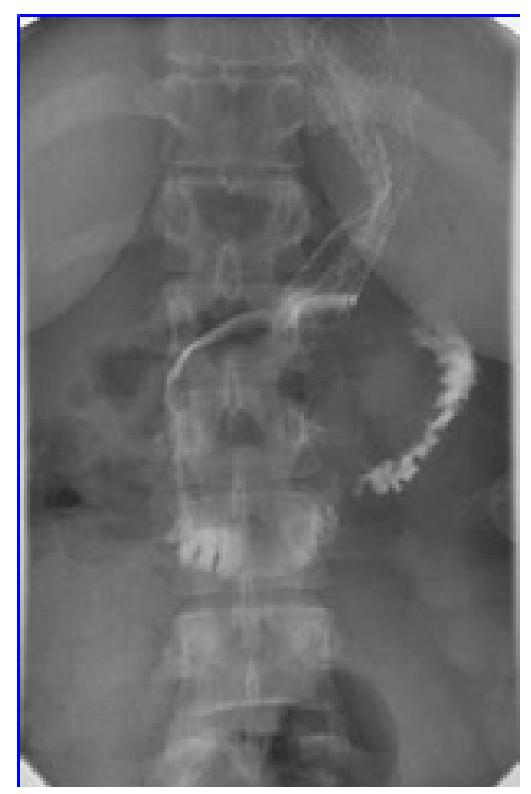

Fig 1: Contrast study showing stent deployed treatment for proximal staple-line leak with partial obstruction at mid-aspect gastric sleeve. Note there is bending in the stent at its midpoint due stricture in gastric sleeve.

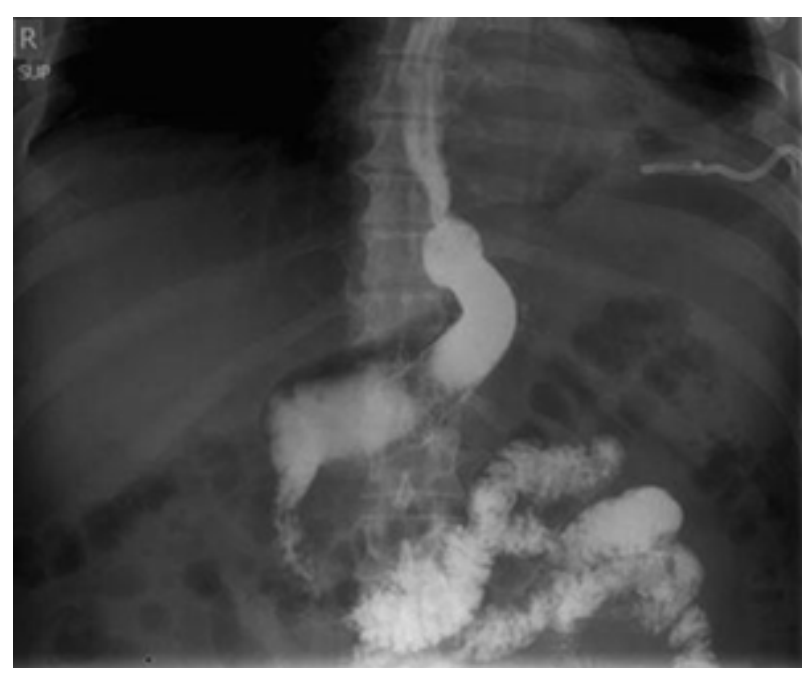

Fig 2: One week after stent deployment upper gastrointestinal contrast study showing good contrast passing freely from the esophagus through stent into gastric antrum with No evidence proximal leak. There is percutaneous drain placed to drain subphrenic collection. 


\section{Results}

The mean age of patients within endoscopy group was $33.83 \pm 8.1$ years with majority of them males $(60 \%)$ and mean BMI $45.14 \pm 6.7 \mathrm{~kg} / \mathrm{m} 2$. Almost $27 \%$ of patients had diabetes and hypertension, 20\% had hypertension and 26.7 had diabetes. The mean age of patients within surgery group was (35.4 \pm 702 ) $44.58 \pm 6.9$ years with majority of them males (53.3\%) and mean BMI $44.58 \pm 6.9 \mathrm{~kg} / \mathrm{m} 2$. Twenty-percent of patients had diabetes, $13.3 \%$ had diabetes plus hypertension, and $13.3 \%$ had hypertension. There was no statistically significant association between type of management and age $(p=0.86)$, BMI $(p=0.15)$, gender $(p=0.73)$, comorbidities $(p=0.48)$. (Table 1$)$.

Mean interval between surgery and leak in endoscopy group was $4.5 \pm 2.7$ days (majority occurring within 4-7 days) and most common site of leak was GE junction (80\%). Mean interval between surgery and leak in surgery group was 4.6 \pm 2.2 days; while most common site leak, GE junction (73.3\%). There was no statistical significance associated between both groups. (Table 2).

Table 1: Relation between demographic characteristics of included patients and type of management

\begin{tabular}{|c|c|c|c|}
\hline Variables & $\begin{array}{l}\text { Endoscopy Group } \\
\qquad(\mathbf{N}=15)\end{array}$ & $\begin{array}{l}\text { Surgery Group } \\
\qquad(\mathbf{N}=\mathbf{1 5})\end{array}$ & P-value \\
\hline $\begin{array}{l}\text { Age in years } \\
\text { Mean } \pm S D \\
\text { Median (Range) }\end{array}$ & $\begin{array}{l}33.83 \pm 8.1 \\
34(21-40)\end{array}$ & $\begin{array}{c}35.4 \pm 702 \\
35(25-48)\end{array}$ & 0.86 \\
\hline $\begin{array}{l}\mathbf{B M I} \text { in } \mathbf{K g} / \mathbf{m}^{\mathbf{2}} \\
\text { Mean } \pm S D \\
\text { Median (Range) }\end{array}$ & $\begin{array}{l}45.14 \pm 6.7 \\
45(24-67)\end{array}$ & $\begin{array}{l}44.58 \pm 6.9 \\
44(33-59)\end{array}$ & 0.15 \\
\hline Gender & No (\%) & No (\%) & \\
\hline Male & $9(60 \%)$ & $8(53.34 \%)$ & 0.73 \\
\hline Female & $6(40 \%)$ & $7(46.75 \%)$ & \\
\hline Comorbidity & No (\%) & No $(\%)$ & \\
\hline DM & $4(26.7 \%)$ & $3(20 \%)$ & \\
\hline HTN & $3(20 \%)$ & $2(13.33 \%)$ & 0.48 \\
\hline HTN DM & $4(26.7 \%)$ & $2(13.33 \%)$ & \\
\hline
\end{tabular}

Table 2: Interval between surgery and leak in days and site of Leak in both groups

\begin{tabular}{|c|c|c|c|}
\hline Variables & $\begin{array}{l}\text { Endoscopy Group } \\
(\mathbf{N}=15)\end{array}$ & Surgery Group ( $\mathbf{N}=15)$ & P-value \\
\hline $\begin{array}{l}\text { Interval between sur } \\
\text { days } \\
\text { Mean } \pm S D \\
\text { Median (Range) }\end{array}$ & $\begin{array}{l}4.5 \pm 2.7 \\
5(3-12)\end{array}$ & $\begin{array}{c}4.6 \pm 2.2 \\
4(1-9)\end{array}$ & 0.147 \\
\hline Site of Leak & No (\%) & No $(\%)$ & \\
\hline Proximal (GE junction) & $12(80 \%)$ & $11(73.33 \%)$ & \\
\hline Mid-sleeve (body) & $3(20 \%)$ & $3(20 \%)$ & \\
\hline Distal (antrum) & 0 & $1(6.66 \%)$ & 0.48 \\
\hline
\end{tabular}

Leak closure was achieved in $13(86.66 \%)$ in endoscopy group and only 2 patients required conversion R en Y. Interval between closure of leak and leak control 3.3 \pm 5.5 days. Average hospital stay was $8.3 \pm 4.4$ days. Leak closure achieved in $7(46.6 \%) 13(86.66 \%)$ in surgery group, seven patients required conversion to $R$ en $Y$ either from the start or after failure of direct suturing to control spillage, while one patients died. interval between closure of leak and leak control was $5 \pm 4.3$ days.
With average hospital stay $8.3 \pm 4.4$ days.

There was no statistically significant association between type of management and outcomes $(p=0.51)$. In contrary, there was statistically significant association between type of management and interval for leak control ( $p<0.001)$ and hospital stay $(p=0.012)$. There was no statistically significant association between the site of leak in both groups $(p=0.48)$. (Table 3). 
Table 3: Outcomes of leak included patients in both groups

\begin{tabular}{lccc}
\hline Variables & $\begin{array}{c}\text { Endoscopy Group } \\
(\mathbf{N}=\mathbf{1 5})\end{array}$ & $\begin{array}{c}\text { Surgery Group } \\
\mathbf{( N = 1 5 )}\end{array}$ & P-value \\
\hline Outcomes, No (\%) & $2(13.33 \%)$ & $7(46.6 \%)$ \\
Conversion to R en Y & $13(86.66 \%)$ & $7(46.66 \%)$ & 0.51 \\
Leak closure & 0 & $1(6.66 \%)$ \\
Death & & \\
Interval between intervention and control & $3.33 \pm 5.5$ & $5 \pm 4.3$ \\
of leak in days & $31(28-44)$ & $4(3-18)$ \\
Mean $\pm S D$ & $12(80 \%)$ & $11 \quad(73.33 \%)$ \\
Median (Range) & $3(20 \%)$ & $3(20 \%)$ \\
Proximal (GE junction) & 0 & $1(6.66 \%)$ \\
Mid-sleeve (body) & & $<0.001$ \\
Distal (antrum) & & \\
\hline
\end{tabular}

Short-term complications in endoscopy group was: restenting $(6.55 \%)$, stent migration required repositioning $(26.66 \%)$, sub-phrenic abscess required US drainage (6.66\%). In long-term follow-up, 2 pa- tients (13.33\%) developed stent-related ulcer and a similar number of patients developed stricture. (Table 4).

Table 4: Complications of management of leak included in patients of endoscopy group

\begin{tabular}{lc}
\hline Variables & Endoscopy Group (N =15) \\
\hline Short-term & No (\%) \\
Restenting & $1(6.6 \%)$ \\
Stent migration (reposition) & $4(26.66 \%)$ \\
Sub-phrenic abscess (US drainage) & $1(6.66 \%)$ \\
Long-term, No (\%) & \\
Stent related ulcer & $2(13.33 \%)$ \\
Stricture & $2(13.33 \%)$ \\
\hline
\end{tabular}

Short-term Table 4: Complications of management of leak included in patients of Surgery group.

complications incidence in surgery group: chest infection (13.33\%), DVT (6.66\%), wound infection $(6.66 \%)$, severe vomiting $(6.66 \%)$, sub-phrenic abscess $(6.66 \%)$. In long-term follow-up, 2 patients $(13.33 \%)$ developed stricture.

Table 5: Complications of management of leak included in patients of Surgery group

\begin{tabular}{lc}
\hline Variables & Surgery Group (N =15) \\
\hline Short-term & No (\%) \\
Chest infection & $2(13.33 \%)$ \\
DVT and chest infection & $1(6.66 \%)$ \\
Wound infection & $1(6.66 \%)$ \\
Sever Vomiting, GERD & $1(6.66 \%)$ \\
Sub-phrenic abscess, US drainage & $1(6.66 \%)$ \\
Long-term & No (\%) \\
Stricture & $2(13.33 \%)$ \\
\hline
\end{tabular}

\section{Discussion}

In our study, mean age of included patients was around 35 years with larger part of patients males. Almost two-thirds of patients had at least one co-morbidity. Juza and associates in 2015 surveyed 
results of various techniques in overseeing staple line spill (SLL). In their study one hundred sixty-five patients experienced LSG SLL identified, major part of patients was females $(60 \%)$ with mean age around 37 years.

Depending on the time of beginning, clinical interference, site of release, and radiological appearance, or blended components breaks in staple line can be grouped. Csendes and co-workers in 2005 characterized early, middle of road and late releases those happening 1-4, 5-9 and 10 or more days following surgical procedure respectively. ${ }^{9}$ Walsh found the commonest area of leak was proximal, close to gastroesophagal (GE) junction. ${ }^{13}$

Because of inappropriate stent placement, in our work, some patients in endoscopy group needed restenting $(6.66 \%)$, stent relocation $(26.66 \%)$, sub-phrenic collection (6.66\%). In long follow up, two of our patients $(13.33 \%)$ had stent- related ulcer and another two developed stricture.

In a single institute study -as in our study- Campos and partners in 2016 had twenty-four patients after LSG with staple-line leaks treated with stents and relocation happened in $22 \%$ of all stents inserted. ${ }^{14}$ Endoscopic management of fistulas after sleeve gastrectomy is successful in $85 \%$ of cases. In cases of collections $>5 \mathrm{~cm}$, internal drainage should be tried first as surgical re-intervention before endoscopy delays treatment success. ${ }^{15}$

Antonio Giuliani and his coworkers found good results using endoscopic double-pigtail stent. Successful leak closures by using double pigtail drainage was $83.41 \%$ in their work. They suggested that double-pigtail stent could be a valid approach to manage the leaks following bariatric gastric surgery, with low rate of complications and a good tolerance by patients. ${ }^{16}$

Matthieu and colleagues had 12 patients with a postLSG leak developed a chronic fistula. All patients underwent an open total gastrectomy with an esophagojejunostomy. They stated that salvage open total gastrectomy with an esophagojejunostomy is a well-tolerated and reproducible salvage procedure for cases of a Post Sleeve Gastrectomy Chronic Fistula, when conservative procedures are not possible. ${ }^{17}$

Early re-suturing within the first three days can bring about fruitful results versus re-suturing of breaks after the third day according to studies by Praveenraj and colleagues in 2016. ${ }^{18}$ Seven patients in our study seen by early careful follow up, were treated by direct suturing of leak site with effective leak control. Charalambos and colleagues concluded in their experience that most leaks resulting from antiobesity surgery were successfully managed using nonoperative methods. Gastrointestinal leaks if managed raidly using computed tomography- guided drainage and/or intraluminal stent placement could be effectively controlled. ${ }^{19}$

Blackham and associates in their work found that early transformation of leaking sleeve gastrectomy into Roux-en-Y gastric bypass was particularly helpful in focuses where interventional skill is not promptly accessible. Their argument was that high pressure zone of the pylorus should avoided by bypassing it. ${ }^{20}$ We had 7 patients treated by transformation into Roux-en-Y, either from the start or after failure of direct suturing to control the leak and spillage was effectively controlled in all them.

In our study, mortality rate was $6.7 \%$. Bariatric holes conveys an expanded mortality of $2-10 \%$ in most series. $^{2}$

\section{Data Availability}

Data is available on request. Please contact the corresponding author (hebatharwatdr@gmail.com).

\section{Conflicts of Interest}

The authors declare that there is no conflict of interest regarding the publication of this paper.

\section{Disclosure}

No funders have any role in the design of the study; in the collection, analyses, or interpretation of data; in the writing of the manuscript; or in the decision to publish the results.

\section{Conclusion}

Laparoscopic sleeve gastrectomy (LSG) has turned into a significant methodology in treatment weight control. Treatment alternatives for postoperative holes after bariatric surgery predominantly rely upon timing of spills introduction. Stenting has expanded in past decade related to progressions in innovation for the treatment anastomotic hole in bariatric procedure.in our study We find stenting better choice for spillage patients who are essentially steady. It is less dangerous than surgical procedure. Anyway further investigations are required to reach a solid conclusion.

\section{References}

1. Osland E, Yunus RM, Khan S, et al: Postoperative early major and minor complications in laparoscopic vertical sleeve gastrectomy (LVSG) versus laparoscopic Roux-en-Y gastric bypass (LRYGB) procedures: A meta-analysis and systematic review. Obes. Surg. 2016; 26(10): 2273-2284.

2. Docimo S, Pryor AD: Management Leaks Endoluminal Stents. In: Chand B. (eds) Endoscopy in Obesity Management. Springer, Cham. 2018; DOIhttps://doi.org/10.1007/978-1-4939-16375_16. 
3. Gagner $M$, Buchwald JN: Comparison of laparoscopic sleeve gastrectomy leak rates in four staple-line reinforcement options: A systematic review. Surg. Obes. Relat. Dis. 2014; 10(4): 713-723.

4. Aurora AR, Khaitan L, Saber AA: Sleeve gastrectomy and the risk of leak: A systematic analysis of 4,888 patients. Surg. Endo. 2011; 26: 1509-15.

5. Gonzalez R, Sarr MG, Smith CS, et al: Diagnosis and contemporary management of anastomotic leaks after gastric bypass for obesity. $\mathbf{J}$ Am $\mathbf{C o l l}$ Surg. 2007; 204: 47-55.

6. Sakran N, Goiten D, Raziel A, et al: Gastric leaks after sleeve gasteectomy: A multicenter experience with 2834 patients. Surg Endosc. 2012; 27: 240-5.

7. Nguyen NT, Nguyen X-MT, Dholakia C: The use of endoscopic stent in management of leaks after sleeve gastrectomy. Obes Surg. 2010; 20: 1289-92.

8. Thomopoulos T, Thoma M, Navez B: Roux-en-Y fistulojejunostomy: A new therapeutic option for complicated post-sleeve gastric fistulas, video report. Obes Surg. 2017; 27(6): 1638-9.

9. Csendes $A$, Burdiles $P$, Burgos $A M$, et al: Conservative management of anastomotic leaks after 557 open gastric bypasses. Obes Surg. 2005; 15: 1252-6.

10. Ballesta C, Berindoague R, Cabrera M, et al: Management of anastomotic leaks after laparoscopic Roux-en-Y gastric bypass. Obes Surg. 2008; 18: 623-30.

11. Praveenraj P, Gomes RM, Kumar S, et al: Management gastric leaks after laparoscopic sleeve gastrectomy for morbid obesity- tertiary care experience design managment algorithm. J Min Access Surg. 2016; 12(4): 342-9.

12. Botaitis $S$, Athanasia $M$, Sempachedin $P$, et al:
Prevention Management Staple Line Leaks after Laparoscopic Sleeve Gastrectomy. J Anesth Surg. 2018; 5(1): 95- 102.

13. Walsh C, Karmali S: Management bariatric complications: Review update. World Journal Gastrointestinal Endoscopy. 2017; 7(5): 518.

14. Campos JM, Ferreira FC, Teixeira AF, et al: Sptotomy and balloon dilation to treat chronic leak after sleeve gastrectomy: Technical principals. Obes Surg. 2016; 26(8): 1992-3.

15. Diane Lorenzo, Anne Dutour, Marc Barthet, et al: Endoscopic treatment of fistulas after sleeve gastrectomy: A comparison of internal drainage versus closure (Gastrointest Endosc). 2018; 87: 429-437.

16. Antonio Giuliani, Lucia Romano, Michele Marchese, et al: Endoscopic double pigtail drainage. A systematic review. Surg Obes Relat Dis. 2019; 15: 1414-1419.

17. Matthieu Bruzzi, Richard Douard, Thibault Voron, et al: Open total gastrectomy with Rouxen-Y reconstruction for a chronic fistula after sleeve gastrectomy. Surgery for Obesity and Related Diseases. 2016; 12: 1803-1809.

18. Praveenraj P, Gomes R M, Palanivelu C, et al: Management of gastric leaks after laparoscopic sleeve gastrectomy for morbid obesity: A tertiary care experience and design of management algorithm. J Minimalaccess Surg. 2016; 12(4): 342-349.

19. Charalambos Spyropoulos, Maria-Ioanna Argentou, Theodoros Petsas, et al: Management of gastrointestinal leaks after surgery for clinically severe obesity. Surg Obes Relat Dis. 2012; 8: 609-615.

20. Blackham R, Hamdorf J, Tan J: Acute Conversion Early Sleeve Gastrectomy Leak Roux-en-Y Gastric Bypass. Obes Open Access. 2017; 3(1): doi http://dx.doi.org/10.16966/2380-5528.128. 